\title{
On a Quadratic First Integral for the Charged Particle Orbits in the Charged Kerr Solution ${ }^{\star}$
}

\author{
LANE P. Hughston \\ Department of Physics: Joseph Henry Laboratories \\ Princeton University, Princeton, New Jersey, USA \\ and \\ Division of Mathematics and Mathematical Physics \\ The University of Texas at Dallas, Dallas, Texas, USA
}

Roger Penrose

Department of Mathematics, Birkbeck College, London, England

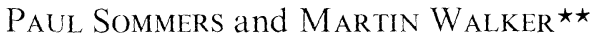

Center for Relativity Theory, The University of Texas at Austin, Austin, Texas, USA

Received May 2, 1972

\begin{abstract}
Associated with the charged Kerr solution of the Einstein gravitational field equation there is a Killing tensor of valence two. The Killing tensor, which is related to the angular momentum of the field source, is shown to yield a quadratic first integral of the equation of the motion for charged test particles.
\end{abstract}

The Hamilton-Jacobi equation for charged particle orbits in the Kerr spacetime ${ }^{1}$ has been found by Carter [1] to be solvable by separation of variables. The separation constant, an expression which is quadratic in the orbital tangent vector, provides in turn a fourth first integral of the equation of motion for charged particles, the other three having been obtained by reason of the two symmetries of the field, and the conservation of the particle rest mass. Walker and Penrose [2] have demonstrated

* The research reported herein has been supported in part by the National Science Foundation, grants GP-8868, GP-20023, and GU-1598; the Air Force Office of Scientific Research, grant 903-67; the National Aeronautics and Space Administration, grant 44-004-001; and the Westinghouse Corporation.

$\star \star$ Present address: Max-Planck-Institute for Physics and Astrophysics, 8000 München 40, Föhringer Ring 6, Germany.

1 Throughout the discussion we refer to the general Kerr family of gravitational fields, with or without either an electromagnetic field [3] or a cosmological constant [1]. 
in an earlier communication how the fourth first integral of the geodesic equation arises from the existence of a tensor $K_{a b}$ which satisfies ${ }^{2}$ $\nabla_{(a} K_{b c)}=0$. In the present communication we show that the quadratic Killing tensor may be used to obtain also Carter's fourth first integral of the equation of motion for charged test particles.

To expedite the discussion, we begin with a simplified proof of the existence of a quadratic Killing tensor for the Kerr spacetime. The proof is based on the following remark: let $o^{A}$ and $l^{A}$ be a normalized spinor dyad whose corresponding null vectors $l^{a}:=o^{A} \bar{o}^{A^{\prime}}$ and $n^{a}:=l^{A} \bar{l}^{A^{\prime}}$ are tangent to congruences of nonshearing null geodesic curves ${ }^{3}$, and let $\phi$ be a complex scalar such that $\phi_{A B}=\phi O_{(A} l_{B)}$ satisfies the vacuum Maxwell equation

$$
\nabla^{A A^{\prime}} \phi_{A B}=0 \text {. }
$$

Then the quantity $\mathrm{X}_{A B}:=\phi^{-3 / 2} \phi_{A B}$ satisfies the twistor equation ${ }^{4}$

$$
\nabla_{(A}^{A^{\prime}} \mathrm{X}_{B C)}=0 \text {. }
$$

To see this, we observe that the dyad component

$$
\left.6 \phi^{3 / 2} o^{A} o^{B} l^{C} \nabla_{(A}{ }^{A^{\prime}}\left[\phi^{-1 / 2} o_{B} l_{C}\right)\right]=o^{A} \nabla_{A} A^{\prime} \phi-2 \phi o^{B} l^{A} \nabla_{A}^{A^{\prime}} o_{B}
$$

of $\nabla_{(A}{ }^{A^{\prime}} \mathrm{X}_{B C)}$ is simply one of the dyad components of $\nabla^{A A^{\prime}}\left[\phi o_{\left(A l_{B}\right)}\right]$, which vanishes by virtue of the Maxwell equation. The component with $o^{A}$ and $l^{A}$ interchanged vanishes for the same reason, and the remaining two components vanish by virtue of the GSF conditions on $O^{A}$ and $l^{A}$.

The converse of the remark above is also true: If $\nabla_{(A}{ }^{A^{\prime}} \mathrm{X}_{B C)}=0$, then the principal spinors of $\mathrm{X}_{A B}$ are $G S F$, and $\left(\mathrm{X}_{C D} \mathrm{X}^{C D}\right)^{-3 / 2} \mathrm{X}_{A B}$ satisfies the Maxwell equation. More generally, the principal spinors of a Killing

2 We use the notation of Penrose [4], as does paper [2]. We also make a number of corrections to paper [2]. Eq. (5) should read $\nabla_{(A}{ }^{A^{\prime}} X_{B C)}=0$. In Eq. (4), the components $g_{03}$ and $g_{30}$ should be $2 m r a R^{-2} \sin ^{2} \theta$; the component $n^{2}$ of Eq. (16) should be $\left(r^{2}-a^{2}-2 m r\right) 2$, and the component $Q^{11}$ in Eq. (17) should be $r^{2}+a^{2}-2 m r$. The equation immediately preceding Eq. (17) should read $Q_{b c}=-2 R^{2} l_{(b} n_{c)}$. Finally, to be consistent with the Battelle conventions for Latin and Gothic indices, the indices in the first line following Eq. (16) should be Latin. The first unnumbered equation after Eq. (17) and the remark following it should read

$$
" \delta_{a}^{a} \delta_{b}^{b} \delta_{c}^{c} \nabla^{a} Q^{b c}=g^{c(a} \nabla_{c} Q^{b c)}-Q^{c(a} \nabla_{c} g^{b c)}
$$

in any coordinate system $x^{a}$, with $\delta_{a}^{a}:=\nabla_{a} x^{\mathrm{a}}$, so that $\nabla_{\mathrm{a}}=\partial / \partial x^{\mathrm{a}}$."

${ }^{3}$ Such spinors will be said to be $G S F$. We notice that the $G S F$ condition $O^{A} O^{B} V_{A A^{\prime}} O_{B}=0$ is preserved under the rescaling $o^{A} \rightarrow \lambda o^{A}$ for an arbitrary complex scalar $\lambda$.

${ }^{4}$ For a conformally flat spacetime, a solution of Eq. (2) describes a symmetric twistor of valence two. A solution of Eq. (2) is generally referred to as a Killing spinor of valence two. 
spinor of arbitrary valence are $G S F^{5}$. In the case of a non-flat vacuum spacetime, this fact would allow us to conclude from the GoldbergSachs theorem [5] that at most two of the principal spinors of a Killing spinor of arbitrary valence are not proportional.

Upon examining the Bianchi identities it may be seen readily that in any vacuum spacetime of type $\{22\}$ with a Weyl spinor $\psi o_{(A} o_{B} l_{C} l_{D)}$ we can construct an electromagnetic test field of the kind we have been considering above: $\phi_{A B}=\psi^{2 / 3} o_{(A} l_{B)}$. Moreover, it is well known that in correspondence with each vacuum spacetime of type $\{22\}$ there exists a parametrized family of type $\{22\}$ solutions of the Einstein-Maxwell equations for which the principal null directions of the electromagnetic field coincide with those of the gravitational field ${ }^{6}$. Here the test electromagnetic fields of interest are already at our disposal. Associated, then, with each of these spacetimes is a Killing spinor of valence two ?

Given the Killing spinor $\mathrm{X}_{A B}$ we define the tracefree symmetric tensor

$$
P_{a b}:=\mathrm{X}_{A B} \overline{\mathrm{X}}_{A^{\prime} B^{\prime}}
$$

As a consequence of Eq. (2), $P_{a b}$ is found to satisfy, as it is shown in paper [2], the conformal Killing equation,

$$
\nabla_{(a} P_{b c)}=P_{(a} g_{b c)}
$$

where $g_{a b}$ is the spacetime metric and where the vector $P^{a}$ is defined by $P^{a}:=\frac{1}{3} \nabla_{b} P^{a b}$. If $P_{a}$ is the gradient of a scalar $P$, then it may easily be verified that the tensor

$$
K_{a b}:=P_{a b}-P g_{a b}
$$

satisfies the Killing equation:

$$
\nabla_{(a} K_{b c)}=0 .
$$

5 Let us consider, for example, an algebraically general Killing spinor $X_{A_{1}} A_{n}$ of valence $n$ :

$$
\begin{array}{ccc}
\nabla_{A^{\prime}\left(A_{1}\right.} \mathrm{X}_{A_{1}} & \left.A_{n}\right) \\
\mathrm{X}_{A_{1}} A_{n} & =0, \\
\sigma_{\left(A_{1}\right.} & \sigma_{\left.A_{n,}\right)} .
\end{array}
$$

Upon transvection $n$ times with $\sigma_{A}^{n}$ the twistor equation becomes

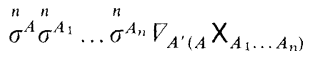

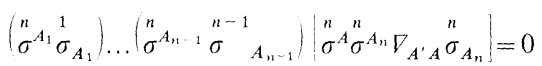

which is seen to be the GSF condition on the congruence defined by $\sigma_{A}^{n}$.

${ }^{6}$ Kinnersley [6] has provided an explicit algorithm for generating the electrification of any type $\{22\}$ vacuum spacetime.

7 The argument given in paper [2] for the existence of a Killing spinor of valence 2 is not quite complete as it stands for the charged Kerr solution, since the proof given would seem to rely on the vacuum Bianchi identities. Our procedure here should clarify that derivation. 
Let us examine the condition that $P_{a}$ be a gradient. This condition may be expressed in a particularly convenient form if we first observe that the stress-energy tensor

$$
\tau_{a b}:=\phi_{A B} \bar{\phi}_{A^{\prime} B^{\prime}}=\phi \bar{\phi}\left[l_{(a} n_{b)}-\frac{1}{4} g_{a b}\right]
$$

of the test electromagnetic field $\phi_{A B}$ is proportional to the conformal Killing tensor $P_{a b}$, and satisfies

$$
\nabla^{a} \tau_{a b}=0
$$

as a consequence of the vacuum Maxwell equation. From these facts it follows that

$$
P_{a}=\frac{1}{3} \nabla^{b} P_{a b}=f^{2} \tau_{a b} \nabla^{b} f=l_{(a} n_{b)} \nabla^{b} f-\frac{1}{4} \nabla_{a} f
$$

where $f:=(\phi \bar{\phi})^{-1 / 2}$. Therefore, the requirement that $P_{a}$ be a gradient is equivalent to the existence of a scalar $h$ such that

$$
l_{(a} n_{b)} \nabla^{b} f=\nabla_{a} h .
$$

We shall now consider explicitly the case of the Kerr spacetime. In the coordinate system of paper [2], the scalar $\phi$ is given by

$$
\phi=(r-i a \cos \theta)^{-2}
$$

so that $f=r^{2}+a^{2} \cos ^{2} \theta$. A simple computation shows that

$$
l_{(a} n_{b)} \nabla^{b} f=\nabla_{a}\left(\frac{1}{2} r^{2}\right) .
$$

Thus we establish that the Kerr spacetime admits a Killing tensor of valence two ${ }^{8}$.

The equation of motion of a test particle with charge $\varepsilon$, moving under the influence of an electromagnetic field $F_{a b}$, may be written

$$
t^{c} \nabla_{c} t^{a}=\varepsilon t^{c} F^{a}{ }_{c},
$$

where $t^{a}$ is tangent to the orbit of the particle. As in the case of an affinely parametrized geodesic orbit, as would be given by $\varepsilon=0$, the equation of motion admits $g^{a b} t_{a} t_{b}$ as a first integral since

$$
t^{c} \nabla_{c}\left(g^{a b} t_{a} t_{b}\right)=2 t^{b} t^{c} \nabla_{c} t_{b}=2 \varepsilon t^{b} t^{c} F_{b c}=0 .
$$

Suppose that $g_{a b}$ admits a one parameter group of isometries generated by a Killing vector $\xi^{a}$, so that $\mathscr{L}_{\xi} g_{a b}=0$. A first integral may be constructed from $\xi^{a}$ provided that the electromagnetic field is also invariant under the action of the group. In fact, we may demonstrate that $\mathscr{L}_{\xi} F_{a b}=0$ is the necessary and sufficient condition that there exist a

8 We comment, incidentally, that our analysis is more generally valid for the charged Kerr-NUT spacetimes $[1,7]$ wherein the Killing tensor assumes the form

$$
K_{a b}=(l-a \cos \theta)^{2} l_{(a} n_{b)}+r^{2} m_{(a} \bar{m}_{b)}
$$

where $l$ is the $N U T$ parameter. 
scalar $\alpha$ such that

$$
t^{c} \nabla_{c}\left(\xi^{a} t_{a}+\varepsilon \alpha\right)=0 .
$$

Using the equation of motion (4) we find Eq. (5) to be satisfied if and only if

$$
\nabla_{a} \alpha+F_{a}^{c} \xi_{c}=0
$$

for which we have the integrability condition

$$
\nabla_{[a}\left(F_{b] c} \xi^{c}\right)=0 \text {. }
$$

Then, using Maxwell's equations in the form

$$
2 \nabla_{[a} F_{b] c}+\nabla_{c} F_{a b}=0
$$

we arrive at the desired relation:

$$
\xi^{c} \nabla_{c} F_{a b}+F_{a c} \nabla_{b} \xi^{c}+F_{c b} \nabla_{a} \xi^{c}:=\mathscr{L}_{\xi} F_{a b}=0 .
$$

If a local vector potential $A_{a}$ for $F_{a b}$ be chosen with the gauge condition $\mathscr{L}_{\xi} A_{a}=0$, then $\alpha=\xi^{a} A_{a}$, and the conserved quantity is given by

$$
\xi^{a}\left(t_{a}+\varepsilon A_{a}\right) \text {. }
$$

Thus far our discussion of first integrals has been valid in general. We restrict our attention now to those spacetimes which admit a Killing spinor $\mathrm{X}_{A B}$, and ask under what conditions we can construct from $\mathrm{X}_{A B}$ a quadratic first integral for the orbits defined by Eq. (4). First we consider the case in which the orbit is null $^{8}$. If the tangent to the orbit is written $t^{a}:=\lambda^{A} \bar{\lambda}^{A^{\prime}}$, then the equation of motion (4) assumes the form

$$
\begin{gathered}
\bar{\lambda}_{A^{\prime}}\left(t_{c} \nabla^{c} \lambda_{A}+\varepsilon \phi_{A B} \lambda^{B}\right)+\lambda_{A}\left(t_{c} \nabla^{c} \bar{\lambda}_{A^{\prime}}+\varepsilon \bar{\phi}_{A^{\prime} B^{\prime}} \bar{\lambda}^{B^{\prime}}\right)=0 \\
F_{a b}:=\phi_{A B} \varepsilon_{A^{\prime} B^{\prime}}+\bar{\phi}_{A^{\prime} B^{\prime}} \varepsilon_{A B}
\end{gathered}
$$

from which we deduce that

$$
t^{c} \nabla_{c} \lambda_{A}+\varepsilon \phi_{A B} \lambda^{B}=i \omega \hat{\lambda}_{A}
$$

for some real scalar $\omega$. A suitable new choice for the phase of $\lambda_{A}$ serves to eliminate $\omega$. Then the equation of motion becomes

$$
t^{c} \nabla_{c} \lambda_{A}+\varepsilon \phi_{A B} \lambda^{B}=0 \text {. }
$$

It is easy to see that the complex scalar $\mathrm{X}:=\mathrm{X}_{A B} \lambda^{A} \lambda^{B}$ formed from the Killing spinor $\mathrm{X}_{A B}$ is constant along the orbits, $t^{a} \nabla_{a} \mathrm{X}=0$, provided that

$$
\mathrm{X}_{B(A} \phi_{C)}{ }^{B}=0 \text {, }
$$

which is precisely the condition that $\mathrm{X}_{A B}$ and $\phi_{A B}$ be proportional. Therefore, for those spacetimes in which the principal null directions of the electromagnetic field are aligned with those of the Killing spinor

9 The null charged particle orbits are, due to their conformal invariance, of some interest in connection with the theory of twistor quantization [8]. 
we obtain a first integral for the charged null orbits. This condition is fulfilled, of course, for the charged Kerr spacetime.

We now demonstrate the construction of a first integral from the Killing tensor

$$
K_{a b}:=\mathrm{X}_{A B} \overline{\mathrm{X}}_{A^{\prime} B^{\prime}}-P g_{a b}
$$

for the non-null charged particle orbits in the Kerr case. Applying Eqs. (3), (4), and (6) we find that

$$
\begin{aligned}
t^{c} \nabla_{c}\left(K^{a b} t_{a} t_{b}\right)= & 2 \varepsilon t^{a} t^{c} K_{b(a} F_{c)}{ }^{b}=-2 \varepsilon t^{a} t^{c}\left[\mathrm{X}_{A C} \overline{\mathrm{X}}_{B^{\prime}\left(A^{\prime}\right.} \bar{\phi}^{B^{\prime}} C^{\prime}\right) \\
& \left.+\overline{\mathrm{X}}_{A^{\prime} C^{\prime}} \mathrm{X}_{B(A} \phi_{C)}^{B}\right],
\end{aligned}
$$

which vanishes identically due to the proportionality $\mathrm{X}_{A B}=\phi^{-3 / 2} \phi_{A B}$. Thus we obtain the first integral

$$
K^{a b} t_{a} t_{b}
$$

We remark, finally, on the physical meaning of the Killing spinor in Kerr's spacetime. Suppose that from the Killing spinor $\mathrm{X}_{A B}$ we construct the bivector

$$
M_{a b}:=\mathrm{X}_{A B} \varepsilon_{A^{\prime} B^{\prime}}+\overline{\mathrm{X}}_{A^{\prime} B^{\prime}} \varepsilon_{A B} .
$$

Then in the linear approximation to Einstein's theory, this bivector can be shown to be related to the expression for the total angular momentum of the source of the gravitational field ${ }^{10}$.

The authors are grateful to Jürgen Ehlers and Ivor Robinson for their conversation.

\section{References}

1. Carter, B.: Commun. math. Phys. 10, 280 (1968); - Phys. Rev. 174, 1559 (1968).

2. Walker, M., Penrose, R.: Commun. math. Phys. 18, 265 (1970).

3. Newman,E.T., Couch,E., Chinnapared, R., Exton,A., Prakash,A., Torrence, R.: J. Math. Phys. 6, 918 (1965).

4. Penrose,R.: Structure of space-time. In: De Witt,C., Wheeler,J.A. (Eds.): Battelle Rencontres. New York: W. A. Benjamin Inc. 1968.

5. Goldberg, J., Sachs, R. K.: Acta Phys. Polon. 22, 13 (1962).

6. Kinnersley, W. M.: Type D gravitational yields (unpublished thesis, California Institute of Technology), (1969).

7. Miller,J.G.: Global analysis of the Kerr-Taub-NUT metric (Princeton University preprint) (1972).

8. Penrose, R., MacCallum, M.A.H.: Twistor theory: An approach to the quantization of fields and space-time (Notes on lectures delivered at the Institute of Theoretical Astronomy, Cambridge) (1972).

Prof. R. Penrose
Department of Mathematics
Birkbeck College
London, England

${ }^{10}$ This last remark can be made in a more concise way in the language of twistors, to which, in the linearized theory, Killing spinors have an especially close relation. 